\title{
Application of genome engineering for treatment of retinal diseases
}

\author{
Dong Hyun Jo ${ }^{1}$ E Jeong Hun Kim ${ }^{1,2,3}$, * \\ ${ }^{1}$ Fight against Angiogenesis-Related Blindness (FARB) Laboratory, Clinical Research Institute, Seoul National University Hospital, Seoul \\ 03080, Departments of ${ }^{2}$ Biomedical Sciences, ${ }^{3}$ Ophthalmology, Seoul National University College of Medicine, Seoul 03080, Korea
}

Genome engineering with clustered regularly interspaced short palindromic repeats (CRISPR) system can be used as a tool to correct pathological mutations or modulate gene expression levels associated with pathogenesis of human diseases. Owing to well-established local administration methods including intravitreal and subretinal injection, it is relatively easy to administer therapeutic genome engineering machinery to ocular tissues for treating retinal diseases. In this context, we have investigated the potential of in vivo genome engineering as a therapeutic approach in the form of ribonucleoprotein or CRISPR packaged in viral vectors. Major issues in therapeutic application of genome engineering include specificity and efficacy according to types of CRISPR system. In addition to previous platforms based on ribonucleoprotein and CRISPRassociated protein 9 derived from Campylobacter jejuni, we evaluated the therapeutic effects of a CRISPR RNA-guided endonuclease derived from Lachnospiraceae bacterium ND2006 (LbCpf1) in regulating pathological angiogenesis in an animal model of wet-type age-related macular degeneration. LbCpf1 targeting Vegfa or Hif1a effectively disrupted the expression of genes in ocular tissues, resulting in suppression of choroidal neovascularization. It was also notable that there

*Corresponding author. E-mail: steph25@snu.ac.kr

https://doi.org/10.5483/BMBRep.2018.51.7.130

Received 4 June 2018

Keywords: Clustered regularly interspaced short palindromic repeats, Genome engineering, Intraocular injection, Retinal diseases, Ribonucleoprotein

Abbreviations: AAV, adeno-associated virus; Cas9, CRISPR-associated protein 9; CRISPR, clustered regularly interspaced short palindromic repeats; HDR, homology-directed repair; indel, insertion and deletion; LbCpf1, CRISPR RNA-guided endonuclease derived from Lachnospiraceae bacterium ND2006; NHEJ, non-homologous end joining

Perspective to: Koo, T. et al., 2018, CRISPR-LbCpf1 prevents choroidal neovascularization in a mouse model of age-related macular degeneration, Nature Communications, 9, 1855. doi: 10.1038/ s41467-018-04175-y. were no significant off-target effects in vivo. [BMB Reports: Perspective 2018; 51(7): 315-316]

Genome engineering is a practical and specific tool to disrupt the expression of genes associated with pathogenesis of diseases. It is also a tool to correct disease-related mutations. Among three genome engineering tools (zinc finger nuclease, transcription activator-like effector nuclease, and clustered regularly interspaced short palindromic repeats [CRISPR]), CRISPR system has advantages as a therapeutic approach over the other two in that it is easier to prepare it for targeting various sequences in the genome. Cell penetration tools and viral vectors such as adeno-associated virus (AAV) can also facilitate the action of CRISPR system in cells. In order to demonstrate the therapeutic potential of genome engineering technology in vivo, we have previously demonstrated that Lipofectamine-assisted delivery of CRISPR/CRISPR-associated protein 9 (Cas9) (Kim et al (2017) Genome Res 27, 419-426) and Cas9 derived from Campylobacter jejuni (CjCas9) packaged into AAV plasmids (Kim et al (2017) Nat Commun 8,14500 ) are feasible ways to administer the CRISPR system.

In the application of genome engineering technology, one of the most prevalent concerns is the selection of delivery tools. In large, there are two types of therapeutic approaches using the genome engineering machinery: ex vivo and in vivo. The ex vivo approach is composed of the following steps: cell extraction from the body, manipulation, and reinjection into the bloodstream. In the process, CRISPR system can be applied in the form of ribonucleoprotein or with the help of viral vectors. On the other hand, the in vivo approach employs delivery of the genome engineering machinery to target organs through the bloodstream or directly.

The eye is an appropriate target for innovative treatment options including gene and cell therapy. There are wellestablished protocols for intravitreal and subretinal administration based on several clinical trials on AAV-mediated gene delivery and implantation of retinal pigment epithelial cells from embryonic or induced pluripotent stem cells. It is anticipated that genome engineering for treatment of ocular diseases can be performed based on these local administration routes. Recently, the Food and Drug Administration of the 
United States has approved voretigene neparvovec (Luxturna, Spark Therapeutics) for AAV-mediated delivery of RPE65 gene by subretinal administration for patients with retinal dystrophy. Our previous preclinical studies have effectively demonstrated therapeutic effects of genome engineering on mouse models of retinal diseases after intraocular administration of the genome engineering machinery (Kim et al (2017) Genome Res 27, 419-426; Kim et al (2017) Nat Commun 8, 14500).

In the study of Koo et al. (2018) (Nat Commun 9,1855), the utility of a CRISPR RNA-guided endonuclease derived from Lachnospiraceae bacterium ND2006 (LbCpf1) in the regulation of pathological angiogenesis from choroidal vasculature was investigated in the eye after intravitreal injection of AAV which encoded LbCpf1 targeting Vegfa and Hif1a. Although hypoxiainducible factor- $1 \alpha$ is a principal regulator of pathological angiogenesis in the retina (Kurihara et al (2012) J Clin Invest $122,4213-4217)$, it is undruggable for a monoclonal antibody with high specificity in that it is an intracellular molecule. Genome engineering adds up our armamentarium against diseases by modulating the expression of genes that encode intracellular proteins with clinical impacts. Frequencies of small insertions and deletions (indels) are primary indicators of the efficacy of genome engineering technology. LbCpf1 targeting Vegfa and Hif1a achieved considerable indel frequencies in ocular tissues which effectively induced downregulation of vascular endothelial growth factor and reduced the formation of choroidal neovascular membranes in vivo.

Another concern for therapeutic application of genome engineering machinery is the occurrence of off-target effects or unwanted targeting of off-target sites. To monitor these, we first performed nuclease-digested whole genome sequencing (Digenome-seq) after digesting cell-free mouse genomic DNA with LbCpf1 ribonucleoprotein complexes targeting Vegfa and Hif1a (Kim et al (2015) Nat Methods 12, 237-243). Surprisingly, there was only one off-target site with LbCpf1 targeting Vegfa. However, the one targeting Hif1a did not have any cleaved sites other than the on-target site. Targeted deep sequencing also demonstrated that AAV encoding LbCpf1 targeting to
Vegfa induced no detectable off-target indels in retinal pigment epithelial tissues and one in retinal tissues at a frequency of $0.17 \pm 0.02 \%$. Further targeted deep sequencing for one potential site which differed from on-target sequences by up to four nucleotides detected no off-target indels in either ocular tissue. All these data suggested the high specificity of LbCpf1 administered in vivo (Diagram 1).

The genome engineering machinery induces double-stranded breaks at on-target sites that are repaired by non-homologous end joining (NHEJ) for indels and homology-directed repair (HDR) with template-based recovery of wild-type sequences. In therapeutic application, NHEJ approach can disrupt the expression of genes associated with disease progression and cut sequences with mutations inherited in an autosomaldominant pattern. On the other hand, the HDR approach employs the genome engineering technology to correct sequences with mutations with any inheritance pattern. Limits in cargo sizes of viral vectors confine the applicability of conventional gene therapy against genetic diseases for which sizes of therapeutic genes are larger than the limits. In this context, it is logical to utilize genome engineering technology in addition to conventional treatment options to treat patients with diseases. Further translational research on genome engineering will help us realize its therapeutic application in clinical settings.

\section{ACKNOWLEDGEMENTS}

This work was supported by the Bio \& Medical Technology Development Program of the National Research Foundation funded by the Korean government, MSIP (NRF-2015M3A9E 6028949 to J.H.K.), Development of Platform Technology for Innovative Medical Measurements funded by Korea Research Institute of Standards and Science (KRISS - 2018 - GP20180018 to J.H.K.), and the Basic Science Research Program through the National Research Foundation of Korea (NRF) funded by the Ministry of Education (2017R1A6A3A04004741 to D.H.J.). 